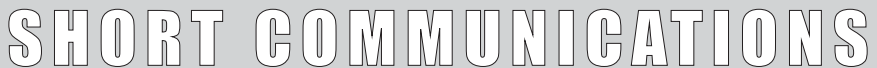

\section{Epidemiological evaluation of sporadic cases of Norovirus infection in comunitary and hospitalized patients}

\author{
Sara Giordana Rimoldi, Cristina Pagani, Alessandra Lombardi, Elena Molteni, \\ Carla Bossi, Claudia Tonielli, Mariarita Gismondo \\ Unità Operativa Complessa di Microbiologia, Azienda Ospedaliera Polo Universitario L. Sacco di Milano
}

Key Words: Norovirus, sporadic cases, epidemiology

Epidemiologia di casi sporadici di gastroenteriti sostenute da Norovirus in pazienti comunitari e nosocomiali affetti da gastroenterite

\section{SUMMARY}

Surveillace of viral gastoenteritis infections is very poor in Italy, even if starting from 2004 Norovirus became one of the most causative agent of infections in all the seasons.

The aim of our study was to evaluate the isolation of Norovirus both in hospitalizes patients and communitary patients. From October 2006 to March 2008 we examined 400 samples. Our results showed only I5 sporadic cases in pediatric, HIV comunitary patients.

These cases were analyzed by using an ELISA screening (Biopharm) and the results were confirmed with real time PCT (Argene).

\section{INTRODUZIONE}

Nel nostro Paese la mancanza di un sistema organico di sorveglianza delle patologie acute del tratto gastroenterico fa sì che la loro epidemiologia, dovute per il $70 \%$ a un'eziologia virale, non sia ad oggi ben conosciuta $(3,5,10)$.

I Norovirus (NoV) sono al terzo posto tra i più comuni agenti eziologici responsabili capaci di provocare ogni anno negli USA circa 9 milioni di casi di malattia $(4,11)$. In Europa la prima grande epidemia da NoV GII/4 risale al 1995, nelle zone di Inghilterra e Galles. Nel 2002 vaste epidemie hanno coinvolto Germania e Olanda nel mese di gennaio, Francia e Spagna nel mese di aprile, Finlandia in giugno ed Ungheria e Danimarca in settembre (8).

L'ultima variante del genotipo GII/4 è stata identificata nel 2006 in Spagna (ceppo 2006b), a seguito di una severa epidemia avvenuta in un istituto residenziale.

In Italia nel 2002 è stata documentata un'epidemia da NoV nella città di Bari per consumo di frutti di mare contaminati; nel 2006-2007 casi sporadici nella provincia di Parma (13) e di Bergamo.

Conosciuti come piccoli virus a RNA con struttura sferica e privi di envelope, hanno un genoma a singolo filamento di RNA a polarità positiva, poliadenilato, con una lunghezza compresa tra i 7.400 e i 7.700 nucleotidi e costituito da 3 ORFs (6): ORF1 codifica per una grande poliproteina che promuove il clivaggio proteolitico, l'ORF2 e l'ORF3 che codificano per due proteine strutturali, rispettivamente VP1 e VP2, rispettivamente proteina minore e maggiore del capside (9).

Sono suddivisi in 5 genogruppi in base alla sequenza nucleotidica della RNA polimerasi RNA dipendente e delle regioni del capside; di questi, solo i genogruppi I, II e IV sono associabili a gastroenteriti umane (9) di cui sono noti 7 genotipi NoV GI, 12 genotipi NoV GII e 2 genotipi in NoV G IV (1).

\section{MATERIALI E METODI}

Per questo studio, condotto in un periodo compreso tra Ottobre 2006 e Marzo 2008, sono stati utilizzati 400 campioni fecali provenienti da sogget-

\section{Corresponding author: Sara Rimoldi}

A.O. Polo universitario "L. Sacco"

Via G.B. Grassi, 74 - 20I57, Milano, Italy

E-mail:rimoldi.sara@hsacco.it 
ti affluenti ai Poliambulatori dell'Azienda Ospedaliera "L. Sacco" $(\mathrm{n}=120,30 \%)$ e da pazienti ricoverati nei reparti di Pediatria $(n=132$, $20 \%)$ e di Malattie Infettive ( $\mathrm{n}=148,37 \%)$.

Requisito di inclusione era la sintomatologia propriamente gastroenterica, la negatività alla coprocoltura, per Salmonella e Shigella, e per Rotavirus e Adenovirus, determinata mediante la ricerca dell'antigene specifico. I campioni fecali raccolti, sono stati dapprima testati mediante uno screening immunoenzimatico Redascreen (R-Biopharm AG, Germany) e successivamente confermati nella loro positività mediante Real time PCR (Argene, Francia).

\section{RISULTATI}

Il presente lavoro si proponeva di valutare il ruolo eziologico dei Norovirus nel determinismo delle gastroenteriti virali.

La ricerca di Antigene NoV è risultata positiva in 15 dei 400 (4\%) campioni, di cui 6 (1.5\%) provenivano dalla Pediatria, $5(1.25 \%)$ dal reparto di Malattie Infettive e 4 (1\%) dai Poliambulatori.

La conferma della presenza dell'RNA mediante real time PCR si è verificata in solo 10 casi.

Dal punto di vista dell'andamento stagionale delle infezioni sostenute da NoV è stato possibile osservare un'aumento numerico dei campioni giunti all'analisi a partire dal mese di Marzo ma le positività si sono verificate nei mesi di Marzo e Aprile 2007 (10) e in Ottobre (2), Novembre (2) e Dicembre 2007 (1).

\section{CONCLUSIONI}

I NoV rappresentano un importante problema di sanità pubblica nel mondo, soprattutto laddove si verifichino clusters epidemici significativi, per via della elevata infettività attraverso acqua e cibi contaminati, per via feco-orale ed aerea, e per la loro incapacità di indurre immunità duratura $(11,8)$. L'assenza di sistemi di coltivazione di coltura in vitro e la mancanza di un modello animale hanno ostacolato per anni le conoscenze sulla reale diffusione dei Norovirus, la cui identificazione si basava, fino agli anni Novanta, essenzialmente sulle tecniche di immunomicroscopia elettronica. La scoperta dell'organizzazione genomica, e la conseguente messa a punto di sistemi diagnostici immunoenzimatici e molecolari, hanno dato un nuovo slancio allo studio delle caratteristiche virologiche ed epidemiologiche dei $\mathrm{NoV}$, ponendo l'attenzione sull'utilità della diagnosi eziologica in tutti i casi sospetti, anche non in presenza di epidemia (casi sporadici), caratterizzati da episodi diarroici, soprattutto in età infantile (13).

Nel presente studio è stato possibile osservare alcuni casi sporadici di infezione da NoV, sia in pazienti pediatrici che in adulti. In particolare sono stati diagnosticati 15 casi mediante il metodo immunoenzimatico e in 10 di questi è stato possibile riscontrare positività per sequenze geniche di RNA virale mediante Real time PCR. E' stato pertanto possibile evidenziare il ruolo eziologico dei Norovirus nel 1.5\% delle manifestazioni gastroenteriche presentate dai pazienti ricoverati nel reparto di Pediatria (6/132), nel 1.25\% (5/148) dei pazienti del reparto di Malattie Infettive e nel $1 \%(4 / 120)$ degli afferenti ai Poliambulatori.

Per quanto concerne l'andamento temporale delle infezioni da NoV, nella prima parte dello studio non sono stati osservati casi positivi, solo a partire dal mese di Febbraio 2007 fino ad Aprile e tra Ottobre e Dicembre. Ciò in parte contrasta con quanto riportato in letteratura, dove la patologia da NoV viene definita come "malattia del vomito invernale"(11, 2, 7).

Per quanto la tendenza di pediatri e medici di famiglia, a fronte di diarrea e dissenteria, sia di attendere la risoluzione della malattia, appare importante poter inserire nella routine di laboratorio metodiche in grado di evidenziare altre infezioni, quali quelle da Norovirus, sia per l'acquisizione di dati epidemiologici che per la definizione diagnostica, al fine di evitare l'uso di rimedi farmacologici costosi e inutili, quali gli antibiotici, di fronte ad infezioni di origine virale.

In conclusione lo studio condotto ha mostrato la presenza di casi sporadici di infezioni sostenute da NoV in fasce d'età diverse in pazienti ospedalizzati e comunitari. La loro ricerca in routine, con indagini di laboratorio mirate, potrebbe quindi contribuire alla diagnosi differenziale delle manifestazioni gastroenteriche, al fine di definire i casi ad eziologia sconosciuta ed attuare misure preventive nei confronti di possibili epidemie, soprattutto in particolari periodi dell'anno.

\section{BIBLIOGRAFIA}

1. Ando, T et al. Genetics classification of "Norwalk like viruses"J.Infect. Dis. 181(suppl.): S336 - S348); 2000.

2. Atmar, RL, Estes MK. Diagnosis non noncultivable gastroenteritis viruses, the human caliciviruse. Clin. Microbiol. Rev. 14:15 - 37; 2001

3. Bern C, Martines J, De Zoyosa I, Glass RI The magnitudo of the global problem of diarrehoal disease: a ten year update. Bull WHO 1982; 70: 705 - 714

4. Blacklow, N.R and H.B. Greenberg.Viral gastroenteritis. N. Engl.J.Med 325: 252-264; 1990

5. Bottinelli GP, Lagravinese D, Ruggeri FM et al. Studio pilota sulle gastroenteriti da virus del gruppo Norwalk a Brindisi. Microbiologia Medica 2001; 16:258-61

6. Glass RI, Kilgore PE, Holman RC, et al. The epidemiology of rotavirus diarrhea in the United States: survelliance and estimates of disease burden. J infect Dis 1996; 174 (suppl 1): S5-11 
7. Green, K.Y., Chanock RM., Kapkian AZ. Human calicivirus, $\mathrm{p}$ 841-874. In D.M. Knipe and P.M. Howley (ed.)., Fields' Virology, 4 ed., vol.1. Lippincott Williams \& Wilkins, Philadelphia, Pa. 2001

8. Loopman B, et al. Increase in viral gastroenteritis outbreacks in Europe and epidemic spread of new Norovirus variant. European Foodborne Viruses Network

9. Kapkian AZ, Hoshino Y, Chanock RM. Rotaviruses. In: Knipe DM, Howley PM, eds. Fields' virology, $4^{\text {th }}$ edn. Philadelphia: Lippincott Williams and Wilkins, 2001: 1787-833. Medical Book Company, 2001, New
York, p 285-343

10. Koopmans M, Von Bonsdorff C, Vinjè J, de Medici D, Monroe S. Foodborne viruses. FEMS Microbiol Rev 2002; 26:187-205

11. E. Owen Caul. Viral gastroenteritis: small round viruses, calicivirus and astroviruses.Part 1 . The clinical and diagnostic perspective. J Clinic Pathol 49: 874-880, 1996

12. Manuale di Virologia medica, seconda edizione Mc Grawhill - Dianzani

13. Medici MC et al. Molecular epidemiology of Norovirus Infections in sposradic cases of viral gastroenteritis. J Med Virol., 2006(78): 1486 - 1492 